\title{
Performance Analysis of Slotted Aloha Protocol in Wireless Cognitive Radio Networks
}

\author{
M. E. Bayrakdar, and A. Calhan
}

\begin{abstract}
With the growing use of frequency based services and devices, the cognitive radio network has become an important technology for the future of wireless networks. However, the number of frequency based network applications for fixed and mobile devices both in licensed, and unlicensed networks also shows a significant increase. In addition to frequency based applications; for efficient use of spectrum in cognitive radio networks, characteristics of medium access techniques is utilized. Among these medium access techniques, slotted Aloha technique is among the most preferred. In our study, simulation of a cognitive radio network environment that consists of primary and secondary users utilizing slotted Aloha medium access technique is performed. The simulation of designed network environment is carried out by using Riverbed software. Upon making end to end delay analysis of the designed network, the results of maximum number of users that could be supported in cognitive radio networks utilizing slotted Aloha technique is observed.
\end{abstract}

Index Terms - Cognitive radio, delay analysis, slotted Aloha.

\section{INTRODUCTION}

$\mathrm{C}$ OGNITIVE radio network is an important technology for the future of wireless networks [1-2]. The use of frequency based services and devices is increasing day by day. However, the number of frequency based applications on licensed and unlicensed networks for both fixed and mobile devices are increasing [2-3]. Similarly, there is growing need for the ISM (Industrial Science Medical) band. Despite constantly growing number of wireless services and devices, the provision of frequency spectrum is known to be a limited resource. Therefore, it is necessary to use the available spectrum band in a flexible and efficient manner [4]. According to the researches, it is seen that the majority of frequency spectrum is allocated to the primary users as fixed. In addition, a small portion of the spectrum is used for urgent applications. However, although some parts of the spectrum at given time intervals is used by primary users, there is some available part of the spectrum that is used partly and rarely [5]. One way to reduce these restrictions is to update the existing license allocation methods. By providing flexible access to frequency bands without harming primary users, spectrum

M. E. Bayrakdar, Computer Engineering Department, Duzce University, 81620, Duzce, Turkey, (e-mail: muhammedbayrakdar@ duzce.edu.tr).

A. Calhan, Computer Engineering Department, Duzce University, 81620, Duzce, Turkey, (e-mail: alicalhan@duzce.edu.tr). access can be provided to secondary users in an opportunistic way. Thus, productivity and efficiency of the frequency spectrum can be well increased [2].

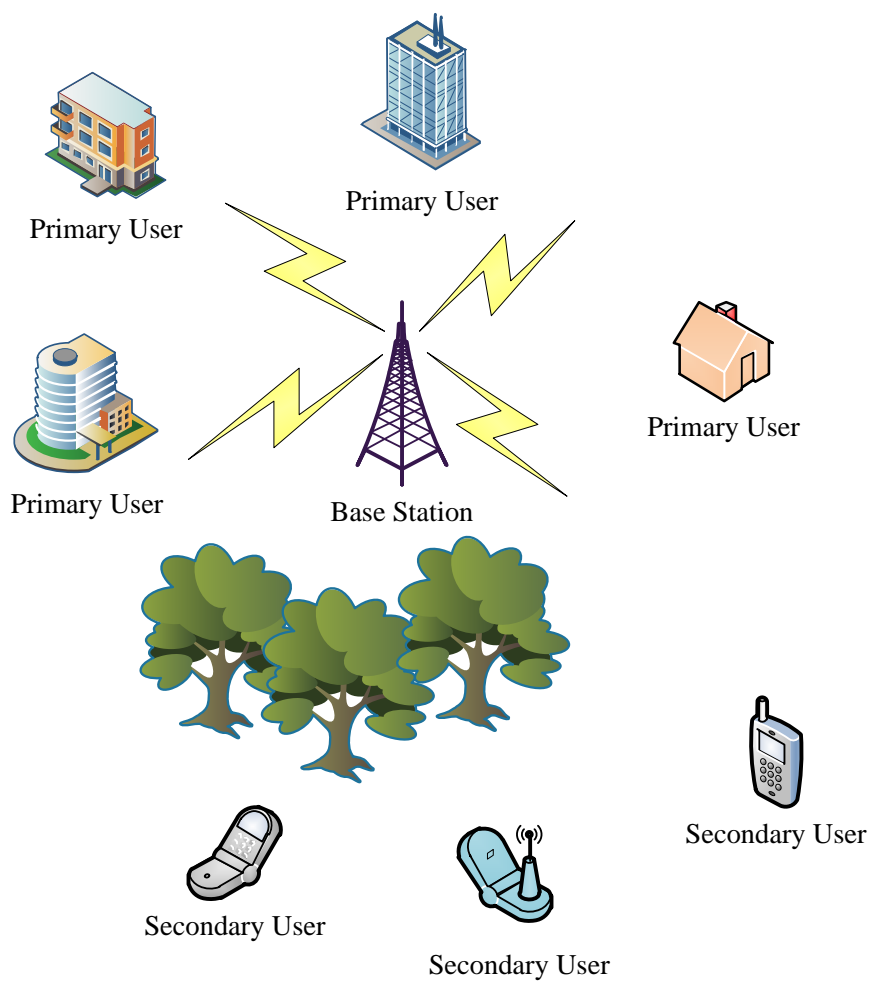

Fig.1. Cognitive radio environment

According to the current communication system policies, there are no flexibility advantages of frequency channels because they work in an allocated way. In contrast, flexible use of frequency channels is provided with cognitive radio technology [3]. Cognitive radio technology is a new technology which aims to be the solution for the future wireless communication technology by ensuring use of the spectrum more efficiently. Cognitive radio is a radio technology that can change the transmission parameters by interacting with its environment. The main tasks of cognitive radio are spectrum detection, spectrum management spectrum mobility and spectrum sharing [2]. The main goal of cognitive radio is to provide the flexibility of the wireless transmission systems with dynamic spectrum access in order to increase spectral efficiency and to optimize transmission performance. The components of the architecture of cognitive radio networks are divided into two separate groups, namely primary and 
secondary networks [5]. Primary networks are licensed networks and they are authorized to use a particular frequency spectrum band. Secondary networks are unlicensed networks, so they can access to the spectrum only through access points opportunistically. Fig. 1 shows a network structure of the primary and secondary users that are located in the same environment.

In our study, a cognitive radio network environment where the primary and secondary users use slotted Aloha technique is considered. Simulation of the designed network environment was carried out using Riverbed software. Delay analysis of the designed network is expressed analytically with mathematical terms and analytical results are seen to be consistent with the simulation results.

\section{SySTEM MOdEl OF THE NETWORK}

In this study, end to end delay analysis of slotted Aloha medium access technique in cognitive radio networks is performed. End to end delay analysis is carried out with simulation scenarios involving different number of primary and secondary users. In the network environment scenarios developed; primary users, secondary users and access points that are serving to these users are existed together.

In Equation (1), analytical model of Slotted Aloha expected delay, $D$ is given [1-3].

$$
D=1+\frac{1-\left(1-\frac{G}{M}\right)^{M-1}}{\frac{G}{M}\left(1-\frac{G}{M}\right)^{M-1}}
$$

where $G$ is total load, and $M$ is number of users [1-3].

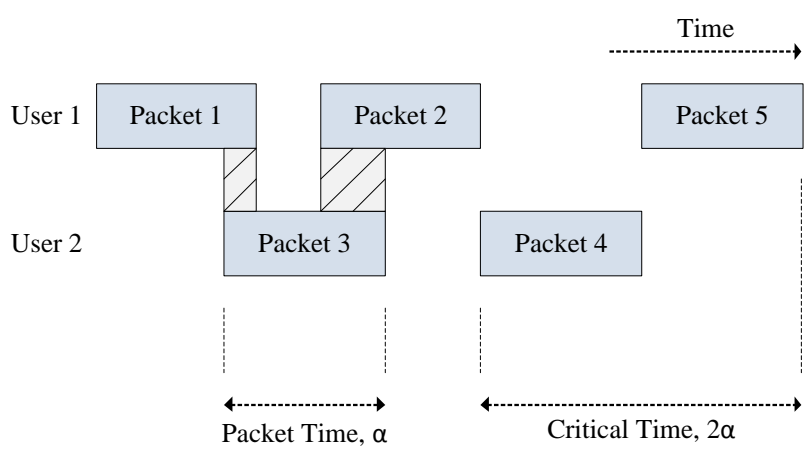

Fig.2. Aloha and slotted Aloha techniques

Slotted Aloha medium access technique is a kind of Aloha protocol that is divided into time periods to reduce the possibility of collisions [6-7]. In the Aloha protocol, direct packet transmission is performed regardless of whether another transmission is present in the environment. The worst part of this protocol is that the collision probability is much higher [89]. In Slotted Aloha medium access technique, when a packet is ready to be transmitted, the process of transmission is performed at the beginning of time periods after waiting until the beginning of the time periods. In this way, packet collisions that may be in the middle of the packet transmissions in Aloha protocol are prevented [10-11]. End to end delay of Slotted Aloha protocol is equal to the elapsed time from the time a packet is produced until reaching the destination.

In Fig. 2, packet transmission of Aloha and slotted Aloha techniques is observed. Here; packet collisions of packets 1, 2, and 3 in Aloha technique is seen. The packets 4, and 5 are transmitted at the beginning of the time period for which they are transmitted according to the characteristics of slotted Aloha techniques.

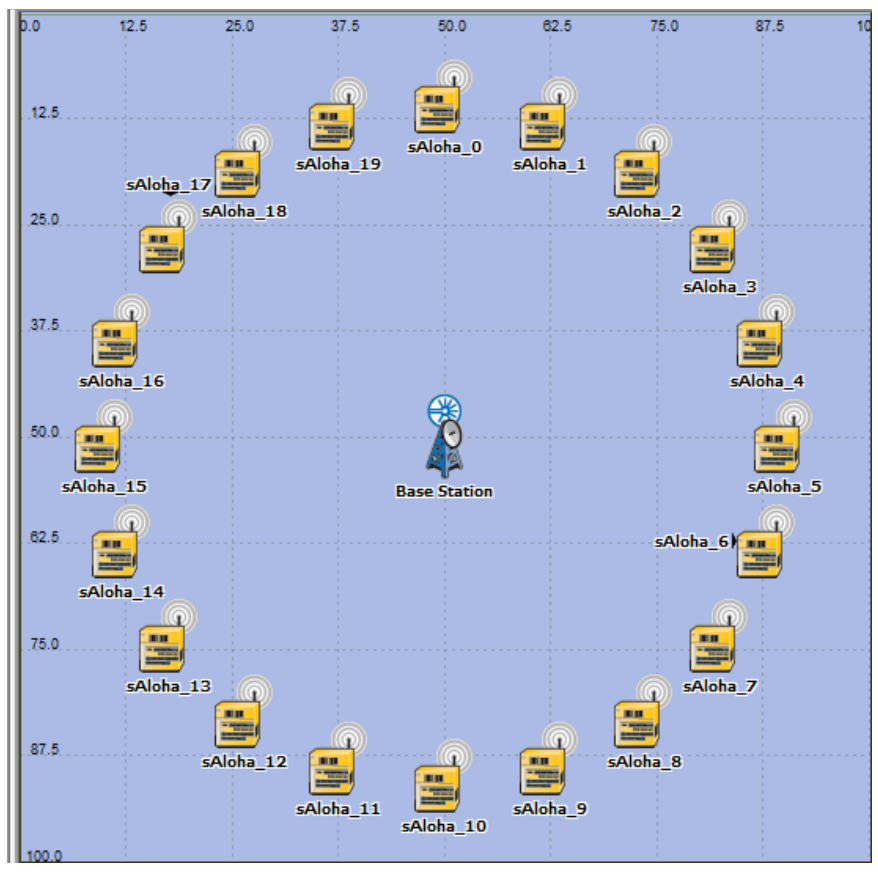

Fig.3. Primary network simulation environment

Fig. 3 shows simulation scenarios designed for primary users performed on Riverbed software. In Fig. 4, combination of cognitive radio network environment where primary and secondary users exist together is seen. Code of each designed node and access point are written in Riverbed simulation software.

Our network model consists of secondary users and base stations in the same communication area with the primary users. Slotted Aloha is exploited as random access scheme by the secondary users. The process of a secondary user in our network is as follows. Each secondary user makes a decision about the time slot usage at the beginning of each time slot. If the time slot is utilized by a primary user, then the secondary user waits for the availability of next time slots. If the time slot is idle, then the secondary user transmits its packet.

In our secondary network model, it is presumed that each secondary user generates packets according to Poisson process. Moreover, the packet length of secondary users is shorter than packet length of primary users because of the spectrum sensing time. 


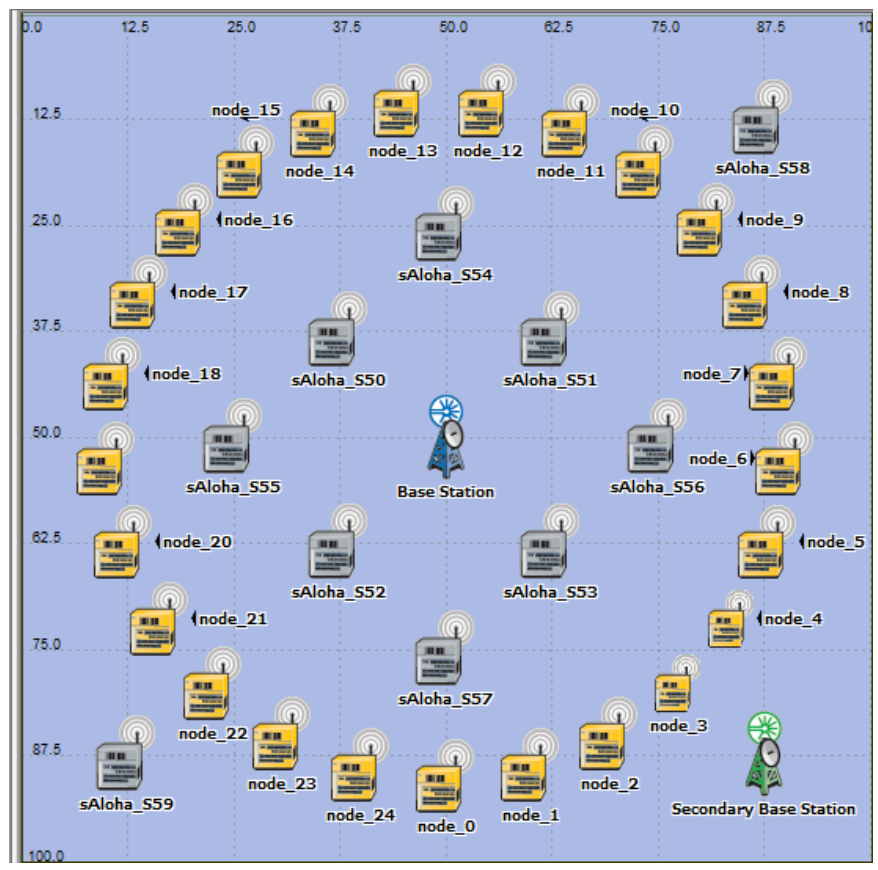

Fig.4. Secondary network simulation environment

\section{Simulation Results OF PRIMARY AND SECONDARY NETWORKS}

This section is devoted to the results obtained concerning the end to end delay and graphics. The obtained numerical results were obtained with the scenarios including different number of users.

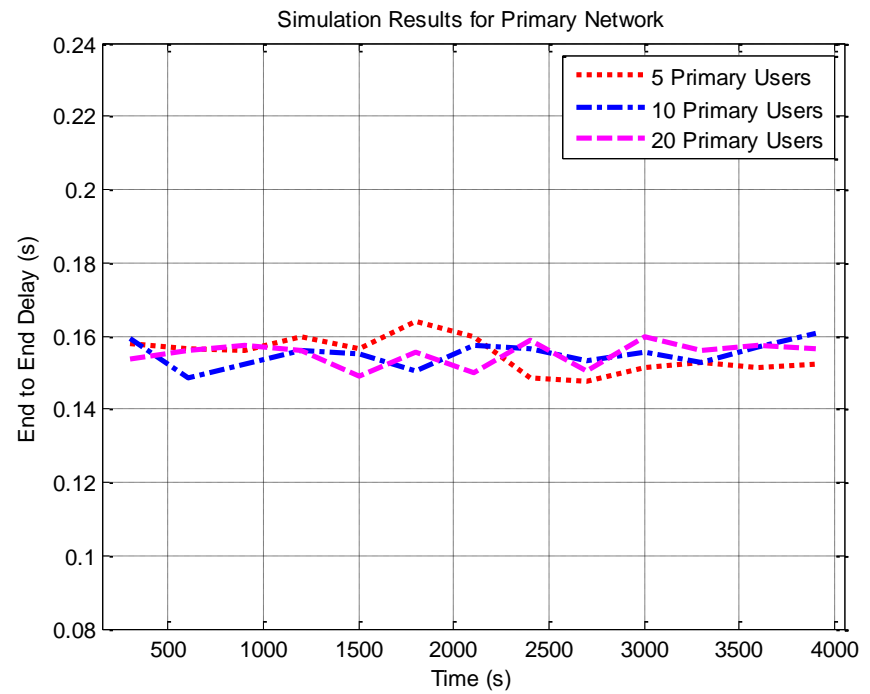

Fig.5. Simulation results of primary network containing 5, 10, and 20 primary users

Fig. 5, and Fig. 6 shows the simulation results for primary networks including only different number of primary users. As seen from the results in Fig. 5, end to end delay results of the primary network including 5,10 or 20 users are pretty close to each other. Here, slotted Aloha technique appears to give very good results up to 20 users. However, the results for the scenarios with 50, and 100 users in Figure 6 is seen to be rather inconsistent. This shows that slotted Aloha technique is not suitable for network environments containing more than 25 users. Besides, overall network performance rather decreases when number of users exceeds 25 because of the collisions.

The Riverbed Modeler software offers some tools such as designing, simulation, and data aggregation [12]. Riverbed Modeler simulation software supports an extensive development environment containing the design of wireless communication networks, and distributed network systems as well [12]. In Riverbed Modeler, performance analysis of a simulation model is evaluated by means of discrete event simulations [12]. Configuration of the network is made in three stage; i.e. network level, node level, and process level. In the network stage, topology of the network is produced. The node stage defines the actions of the node and controls the data flowing in different part of the node. The process stage is characterized by state machines that are formed with states and transitions among them. The source code of Riverbed Modeler simulation software is written on $\mathrm{C}$ programming language [12].

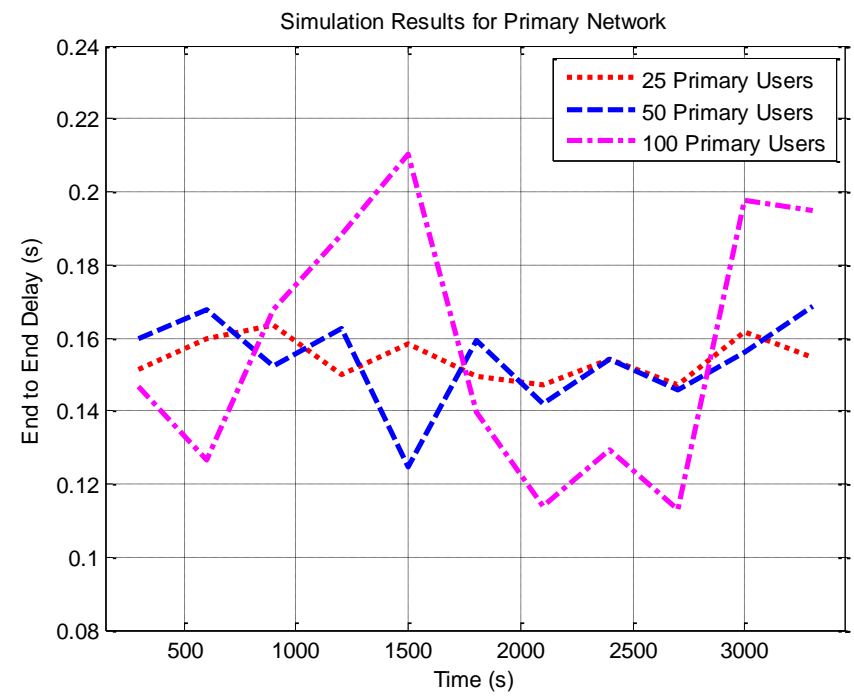

Fig.6. Simulation results of primary network containing 25, 50, and 100 primary users

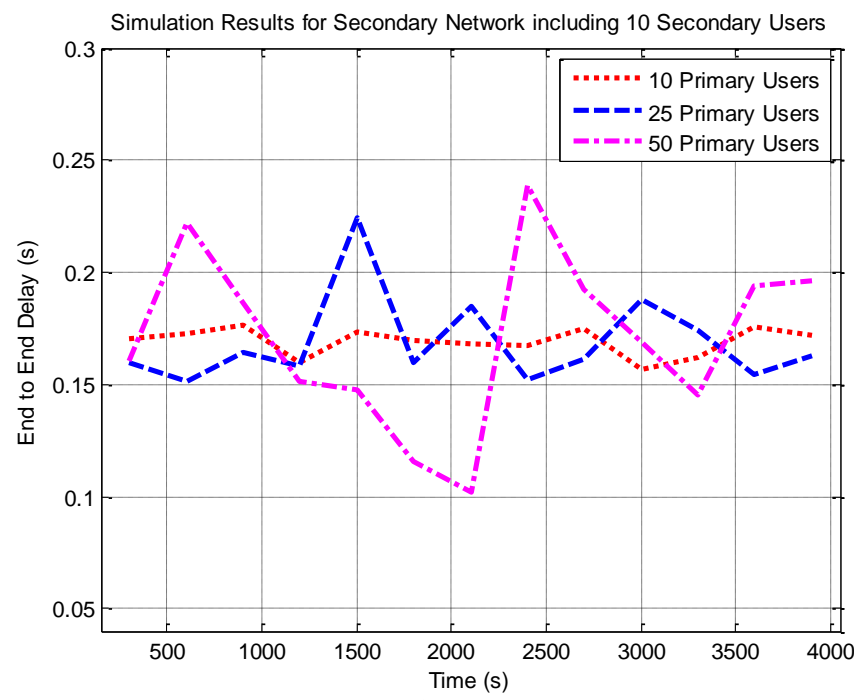

Fig.7. Simulation results of 10 users' secondary network containing 25,50 , and 100 primary users 
In Fig. 7, the simulation results of the secondary network with 10 primary users for different number of secondary users is seen. The graph gives consistent end to end delay results when there are 10 primary users and 10 secondary users in the network structure. End to end delay results of the primary networks including 25, and 50 users are found to be inconsistent. Here, it is seen that cognitive radio networks utilizing slotted Aloha technique with maximum number of 10 primary users and 10 secondary users give appropriate results.

\section{CONCLUSIONS}

In this study, simulation of a cognitive radio network environment which primary and secondary users use slotted Aloha medium access technique that is optimized format of Aloha protocol is carried out. Riverbed software is utilized to perform a simulation of the designed network. Making end to end delay analysis of the designed network, maximum number of users utilizing slotted Aloha technique that is supported in cognitive radio networks has been observed. According to the obtained results, a network structure consisting of the primary users that utilize slotted Aloha technique has been shown to give good results for up to 20 users. In cognitive radio network environment, network infrastructure that includes 10 primary users and 10 secondary users give good results. In subsequent studies, the performance of the secondary users in cognitive radio networks using different medium access techniques are expected to be analyzed.

\section{ACKNOWLEDGMENT}

This work is supported by the Duzce University Scientific Research Projects Office with project number 2015.07.02.329.

The study is selected from National Engineering Research Symposium 2015 (Ulusal Mühendislik Araştırmaları Sempozyumu) UMAS 2015 (Duzce University).

\section{REFERENCES}

[1] A.J. Faria, J. M. C. Brito, "Average Delay in Cognitive Radio Networks using Slotted Aloha", IEEE International Conference on Communications, Computers and Applications, Istanbul, Turkey, 2012.

[2] S. Choe, "Throughput and Delay of Slotted-Aloha based Cognitive Packet Radio Network", IEEE International Conference on Communication Systems, Guangzhou, China, 2008.

[3] H. Takagi, L. Kleinrock, "Throughput-Delay Characteristics of some Slotted-Aloha Multihop Packet Radio Networks", IEEE Transactions on Communications, Vol. COM-33, No. 11, pp. 1200-1207, 1985.

[4] W. Yue, "Output and Delay Processes in a Slotted Aloha Multichannel Packet Radio Network with Capture", IEEE International Phoenix Conference on Computers and Communications, Scottsdale, Arizona, United States of America, 1991.

[5] C. Çiflikçi, A. T. Tuncer, A. T. Özșahin, S. M. Yesbek, "Bilișsel Radyo ve Ortam Erişim Kontrol Katmanı Protokolleri”, Uluslararası İleri Teknolojiler Sempozyumu, Karabuk, Turkey, 2009.

[6] V. Naware, G. Mergen, L. Tong, "Stability and Delay of Finite-User Slotted Aloha with Multi Packet Reception", IEEE Transactions on Information Theory, Vol. 51, No. 7, pp. 2636-2656, 2005.
[7] Y. Yang, T. S. P. Yum, "Delay Distributions of Slotted Aloha and CSMA", IEEE Transactions on Communications, Vol. 51, No. 11, pp. $1846-1857,2003$

[8] G. A. Cunningham, "Delay versus Throughput Comparisons for Stabilized Slotted Aloha", IEEE Transactions on Communications, Vol. 38, No. 11, pp. 1932-1934, 1990.

[9] F. L. Lo, T. S. Ng, T. I. Yuk, "Delay-Throughput Comparison of Single and Multi-Channel Slotted Aloha Networks", IEEE International Conference on Communication Technology, Beijing, China, 1996.

[10] A. Tremeau, N. Borel, "Throughput and Delay Analysis of Two-Tier Slotted Aloha", IEEE Wireless and Optical Communications Conference, Newark, New Jersey, United States of America, 2009.

[11] S. Solak, "Kablosuz Algılayıcı Ağlarda kullanılan MAC Protokollerinin Karşılaştırmalı Başarım Analizi", Yuksek Lisans Tezi, Kocaeli University, Graduate School Of Natural And Applied Sciences, 2008.

[12] Riverbed Modeler 2015. http://www.riverbed.com/products/steelcentral/opnet.html.

\section{BIOGRAPHIES}

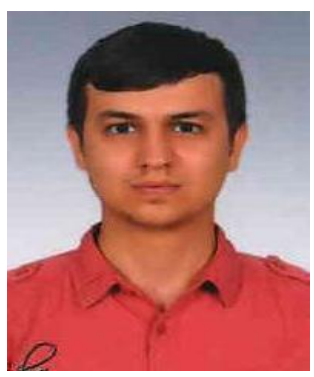

Muhammed Enes BAYRAKDAR was born in Izmit, Turkey in 1988. He received the M.Sc. degree from University of Kocaeli, Turkey in 2013. He studies for Ph.D. degree at Duzce University. His research interests are cognitive radio networks, queuing theory, medium access techniques, wireless communications, and artificial intelligence.

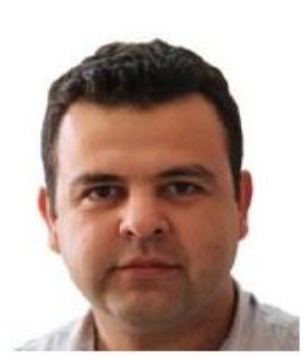

Ali ÇALHAN received the M.Sc. and Ph.D. degrees from University of Kocaeli, Turkey in 2006 and 2011, respectively. His research interests are cognitive radio, wireless heterogeneous networks, wireless communications, and artificial intelligence. 